\title{
Chitinase 3-like-1, a novel regulator of Th1/CTL responses, as a therapeutic target for increasing anti-tumor immunity
}

\author{
Do-Hyun Kim ${ }^{1,2} \mathcal{E} \mathrm{Je}-\mathrm{Min} \mathrm{Choi}^{1,2, *}$ \\ ${ }^{1}$ Department of Life Science, College of Natural Sciences, Hanyang University, Seoul 04763, ${ }^{2}$ Research Institute for Natural Sciences, \\ Hanyang University, Seoul 04763, Korea
}

\begin{abstract}
Chitinase-Like Proteins (CLPs) are an evolutionarily conserved protein which lose their enzymatic activity for degrading chitin macromolecules. Chitinase-3-like-1 (Chi3l1) is a type of CLP that is highly expressed in epithelial cells, macrophages, etc., and is known to have correlations with type 2 inflammation and cancer. Although the increased level of Chi3l1 in the blood was reported in various disease patients, the function of Chi3l1 in adaptive immunity has been totally unknown. Recently, we found that Chi3l1 is expressed in T cells and has a negative regulatory role in T-cell activation and proliferation. A genetic ablation study of Chi3l1 in $\mathrm{T}$ cells showed hyperresponsiveness to TcR stimulation, which increased proliferation and Th1 differentiation. A significant increase of IFN $\gamma$ signaling in Chi3l1-deficient $T$ cells synergistically increased Th1 and CTL functions against melanoma cells in vitro and in vivo. In addition, targeted knockdown by Chi3l1 siRNA complexed with the cell-penetrating peptide dNP2, which showed decreased pulmonary melanoma metastasis with increased infiltration of Th1 and CTL in the lung. This study first suggests that Chi3l1 is a novel regulator of Th1/CTL responses and could be a target for treating cancer to increase tumor immunity. [BMB Reports: Perspective 2018; 51(5): 207-208]
\end{abstract}

*Corresponding author. E-mail: jeminchoi@hanyang. ac.kr

https://doi.org/10.5483/BMBRep.2018.51.5.094

Received 10 April 2018

Keywords: Anti-tumor immunity, Chi311, Chitinase-Like Protein, CTL, Th1

Abbreviations: CAF, Cancer associated fibroblast; Chi311, Chitinase3-like-1; CLP, Chitinase-Like Protein; NSP, Non-signaling peptide; Tfh, Follicular helper T cell

Perspective to: Do-Hyun Kim et al (2018) Regulation of chitinase-3-like- 1 in T cell elicits Th1 and cytotoxic responses to inhibit lung metastasis, Nature Communications 9(503) doi:10.1038 /s41467-017-02731-6
Chitinase is an enzyme that degrades chitin macromolecules, which are long-chain polymers of $\mathrm{N}$-acetylglucosamine, a component of cell walls in fungi and the exoskeletons of arthropods. Surprisingly, several chitinase and chitinase-like proteins (CLPs) have been identified in mammals, and their expression is upregulated in various inflammatory diseases and tumors. Ym1/2 (Chi3l3, Chi3|4) are well-known CLPs as markers of alternative activated macrophages and contributors to allergic diseases. A recent study suggests that these CLPs promote $\gamma \delta$ T-cell mediated-IL-17 production and neutrophilia in parasite infection. Chitinase-3-like protein 1 (Chi3l1), also known as YKL-40 in humans and BRP-39 in mice, is highly expressed in macrophages, epithelial cells, etc. The level of Chi3l1 in the blood has correlations with disease severity in allergic disease, such as asthma, and cancers. Chi3l1 $\mathrm{KO}$ mice were studied in Th2 inflammation models by OVA stimulation or IL-13 transgenic, suggesting Chi3l1 is a marker as well as an inducing factor for Th2 inflammation. Recently, Chi3l1 KO mice were also investigated in a study of pulmonary melanoma metastasis. Melanoma challenge induces Chi3l1 expression, and genetic ablation of Chi3l1 in mice leads to reduced pulmonary melanoma metastasis. More recently, it was reported that a cancer-associated fibroblast (CAF) expressed Chi3l1; secreted Chi3l1 increases tumor progression and M2 macrophage polarization. Collectively, previous studies of Chi3l1 in allergy and cancers suggest that Chi3l1 is a biomarker for inflammatory diseases and cancers that contributes to Th2 inflammation and tumor metastasis. However, its biological roles in adaptive immunity, such as T-cell functions, have been totally unknown.

In a recent study (Kim DH et al (2018) Nat Commun 9, doi:10.1038/s41467-017-02731-6), we reported that Chi3/1 is a novel regulator of Th1/CTL differentiation and could be a target for tumor immunotherapy. We first reported that Chi3/1 is expressed in T cells, and that Chi3l1-deficient CD4 T cells showed increased IFN $\gamma$ and IL-2 production with increased proliferation, suggesting that Chi3l1 could be a negative regulator of $\mathrm{T}$ cell activation. We found that augmented Th1 differentiation without Chi3l1 in T cells could possibly be due to an increased level of pSTAT1 upon IFN $\gamma$ stimuli. In addition, Chi3l1-deficient CD8 T cells secrete cytotoxic materials, such as granzyme $\mathrm{B}$ and perforin. Total

ISSN: 1976-670X (electronic edition)

Copyright (C) 2018 by the The Korean Society for Biochemistry and Molecular Biology

(c) This is an open-access article distributed under the terms of the Creative Commons Attribution Non-Commercial License (http://creativecommons.org/licenses/by-nc/4.0) which permits unrestricted non-commercial use, distribution, and reproduction in any medium, provided the original work is properly cited. 


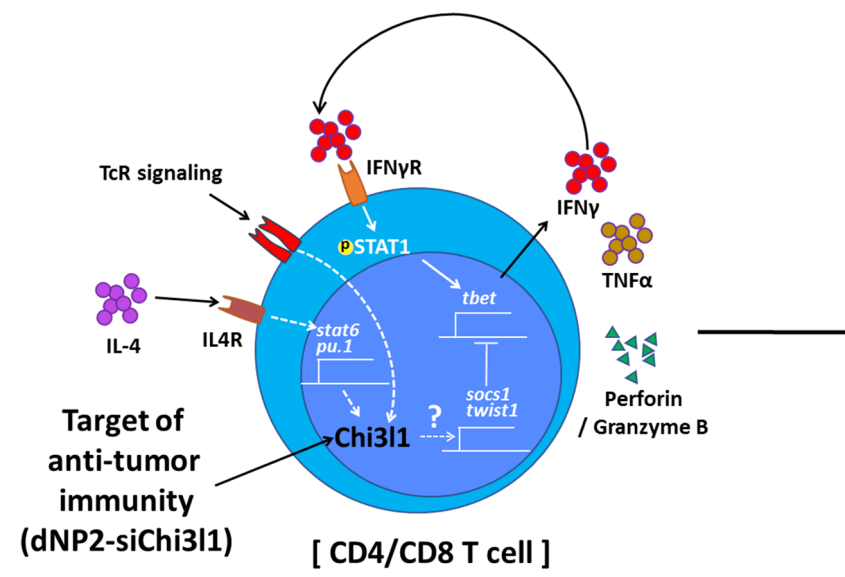

transcriptome analysis by RNA sequencing demonstrated that Chi3l1 deficiency in T cells showed decreased Th1 regulatory genes, such as Twist1 and SOCS1, but there was a significant increase of T-bet, IFNy, ctse, trail, etc., which are anti-tumoral genes. T-cell specific deletion of Chi3l1 in the mice by CD4-Cre showed decreased lung metastasis of melanoma cells with the increased infiltration of IFN $\gamma$, TNF $\alpha$, and GranzymeB-producing T cells in the lung. To prove whether it could be a target for improving tumor immunity, we generated a noncovalent molecular complex of siRNA for Chi3l1 and a cell-penetrating peptide of dNP2, which is a novel cellpermeating peptide we published at Nat Commun 2015. The dNP2 peptide and Chi3l1 siRNA complex (dNP2-siChi3l1) treatment in mice significantly inhibited pulmonary melanoma metastasis with increased T cells producing IFN $\gamma$ and TNF $\alpha$ in the lungs. In conclusion, our studies demonstrate that Chi3/1 is a novel regulator of Th1/CTL differentiation and could be a novel therapeutic target to reject tumor metastasis in the lungs (Diagram 1).

Although we demonstrated that Chi3l1 regulates Th1/CTL response in vivo, its molecular mechanism is still uncertain, because previous studies suggested that secreted Chi3l1 binds
Diagram 1. Suggested hypothesis of Chi3l1 roles in anti-tumoral T-cell immunity. Chi3l1 expression is induced by TCR or IL-4 stimuli in T cells. Chi3l1 might strengthen Th1 inhibitory genes, such as socs1 and twist1, to regulate IFN $\gamma$ sensitivity in $\mathrm{Th} 1$ and CTL. Thus, targeted knockdown of Chi3l1 by dNP2-siChi3l1 elicits Th1 and CTL functions and inhibits tumor growth and metastasis in vivo. to several receptors, such as IL-13R $\alpha 2, \mathrm{CRTH} 2$, and Galectin-3, to initiate a signaling cascade. There are two known isoforms of Chi3l1, a full-length form and a nonsignaling peptide (NSP) form. We suspect that the NSP form of Chi3/1 might be more related to the phenotypes that we observed, because there was no effect of recombinant Chi3l1 treatment on T cells. Therefore, more precise studies regarding these isoforms should be considered to understand their roles in controlling adaptive immunity. This evolutionally conserved chitin-sensing molecule may increase extracellular pathogen immunity, such as Th2 or Tfh response, other than Th1 response, which is our definite future hypothesis to address. In addition, that targeted knockdown of Chi3I1 by siRNA therapy showed increased tumor immunity prompted us to investigate the dNP2-siChi3l1 complex in other tumors, as well as in combination with anti-PD1 or anti-CTLA4 antibodies.

\section{ACKNOWLEDGEMENTS}

This work was supported by a grant from the National Research Foundation (NRF) of South Korea (2017M3A9C 8027972). 